Bull. Chem. Soc. Ethiop. 2021, 35(3), 573-586.

(C) 2021 Chemical Society of Ethiopia and The Authors

ISSN 1011-3924

DOI: https://dx.doi.org/10.4314/bcse.v35i3.9

Printed in Ethiopia

Online ISSN 1726-801X

\title{
NOVEL SYNTHESIS OF PYRAN-3-HYDRAZIDE DERIVATIVES AND THEIR USES TO THE SYNTHESIS HYDRAZIDE-HYDRAZONE, PYRAZOLE AND THIAZOLE DERIVATIVES WITH ANTICANCER ACTIVITIES
}

\author{
Eman M. Samir ${ }^{1}$ and Rafat M. Mohareb ${ }^{2 *}$ \\ ${ }^{1}$ Egyptian Drug Authority (EDA), National Organization for Drug Control and Research \\ (NODCAR), P.O. 29, Cairo, Egypt \\ ${ }^{2}$ Chemistry Department, Faculty of Science, Cairo University, Giza, Egypt
}

(Received March 19, 2021; Revised January 14, 2022; Accepted January 17, 2022)

\begin{abstract}
The multi-component reaction of ethyl acetoacetate with each of malononitrile (3) benzaldehyde (1) in ethanol containing triethylamine gave the ethyl 6-amino-5-cyano-2-methyl-4-phenyl-4H-pyran-3-carboxylate (4). The latter compound reacted with hydrazine hydrate to give the hydrazide derivative $\mathbf{6}$. Compound 6 underwent a series of hetero-cyclization reactions to give pyrzole, hydraide-hydrazone, thiazole derivatives. The produced compounds tested against cancer cell lines six cancer cell lines and showed that compounds 8b, 10b, 11a, 17a, 21 and 24a were the most cytotoxic compounds. Further tests of the latter compounds toward the five tyrosine kinases and Pim-1 kinase showed that compounds 10b, 21 and 24a were the most potent of the tested compounds and compounds 10a, 11a and 17a were of the highest inhibitions toward Pim-1 kinase. The high inhibitions of most of the tested compounds toward the selected cancer cell lines and the tyrosine kinases encourage for future work to be done.
\end{abstract}

KEY WORDS: Hydrazide, Thiophene, Pyrazole, Pyran, Cytotoxicity, Tyrosine kinases

\section{INTRODUCTION}

It was well known that multi-component reactions (MCR) were easy way to mix three or more components together in a one pot reaction to produce high yield products in a very short time. Many compounds were available in literature were found to be easily obtained within a very short reaction time [1-5]. One of the most popular class of compounds obtained the multi-component reaction was the pyran derivatives. Such compounds exhibited wide range of biological activities like antitumor, antibacterial, antibiotic, hypolipidemic, antiallergic, and immune-modulating activities [6]. Among the 6-membered oxygen-comprising heterocycles are the 2-Amino- $4 \mathrm{H}$ pyrans as they show high potencies as antimicrobial and anticancer agents [7,8]. We were concerned within the recent years through heterocyclic transformations using cyclohexan-1,3dione as starting material involved through many multi-component reactions [9-13]. In addition, a number of hydrazide-hydrazone derivatives have been claimed to possess interesting bioactivity such as antibacterial-antifungal, anticonvulsant, antiinflammatory, antimalarial, analgesic, antiplatelets, antituberculosis and anticancer activities [14-21].

In the aim of producing anti-cancer agents, in the present work we were demonstrating the multi-component reactions of ethyl acetoacetate to produce a polysubstituted pyran derivative followed by its conversion into pyran-3-hydrazide followed by its transformation into the corresponding hydrazide-hydrazone derivatives. Based on the different active centers of the latter product, it was involved in many heterocyclization reaction to produce biologically active compounds that can be used as anticancer agents in the future.

\section{RESULTS AND DISCUSSION}

In the present work benzaldehyde (1) underwent a multi-component reaction with ethyl acetoacetate (2) and malononitrile (3) in ethanol containing triethylamine gave the ethyl 6-amino-

*Corresponding author. E-mail: raafat_mohareb@yahoo.com

This work is licensed under the Creative Commons Attribution 4.0 International License 
5-cyano-2-methyl-4-phenyl-4H-pyran-3-carboxylate (4). The structure of the latter product was confirmed on the basis of its analytical and spectral data (see experimental section). Compound 4 reacted with hydrazine hydrate to give the pyran-3-hydrazide derivative $\mathbf{6}$. The reactivity of compound 6 toward different chemical reagents was studied in order to produce biologically active compounds. It was reported that hydrazide-hydrazone derivatives are good candidate as anti-cancer agents [22-24]. This encouraged us to synthesis hydrazide-hydrazone derivatives from compound $\mathbf{6}$. Thus, the reaction of compound 6 with either cyclopentanone (7a) or cyclohexanone (7b) gave the hydrazide-hydrazone derivatives $\mathbf{8 a}$ and $\mathbf{8 b}$, respectively [25]. The structures of the

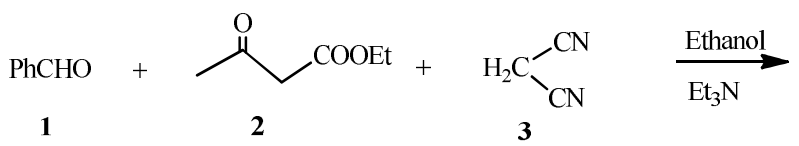<smiles>CCOC(=O)C1=C(C)OC(N)=C(C#N)C1c1ccccc1</smiles><smiles>CC1(NN)CCCCC1</smiles><smiles>CC1=C(C(=O)NN)C(c2ccccc2)C(C#N)=C(N)O1</smiles>

6

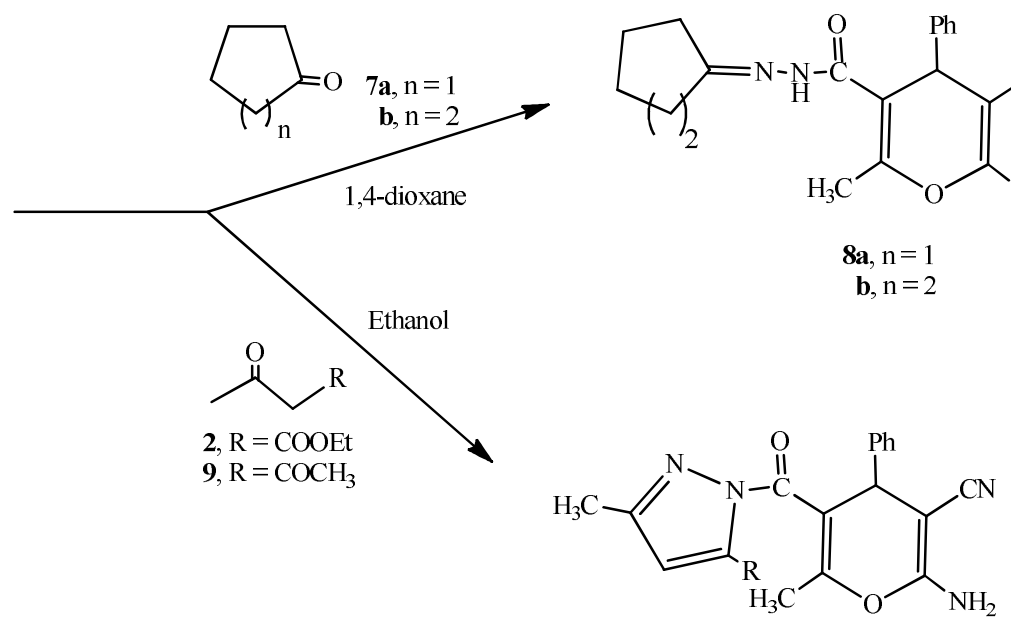

10a, $\mathrm{R}=\mathrm{OH}$

b, $\mathrm{R}=\mathrm{CH}_{3}$

Scheme 1. Synthesis of compounds $\mathbf{4}, \mathbf{6} ; \mathbf{8 a}, \mathbf{b}$ and $\mathbf{1 0 a}, \mathbf{b}$. 


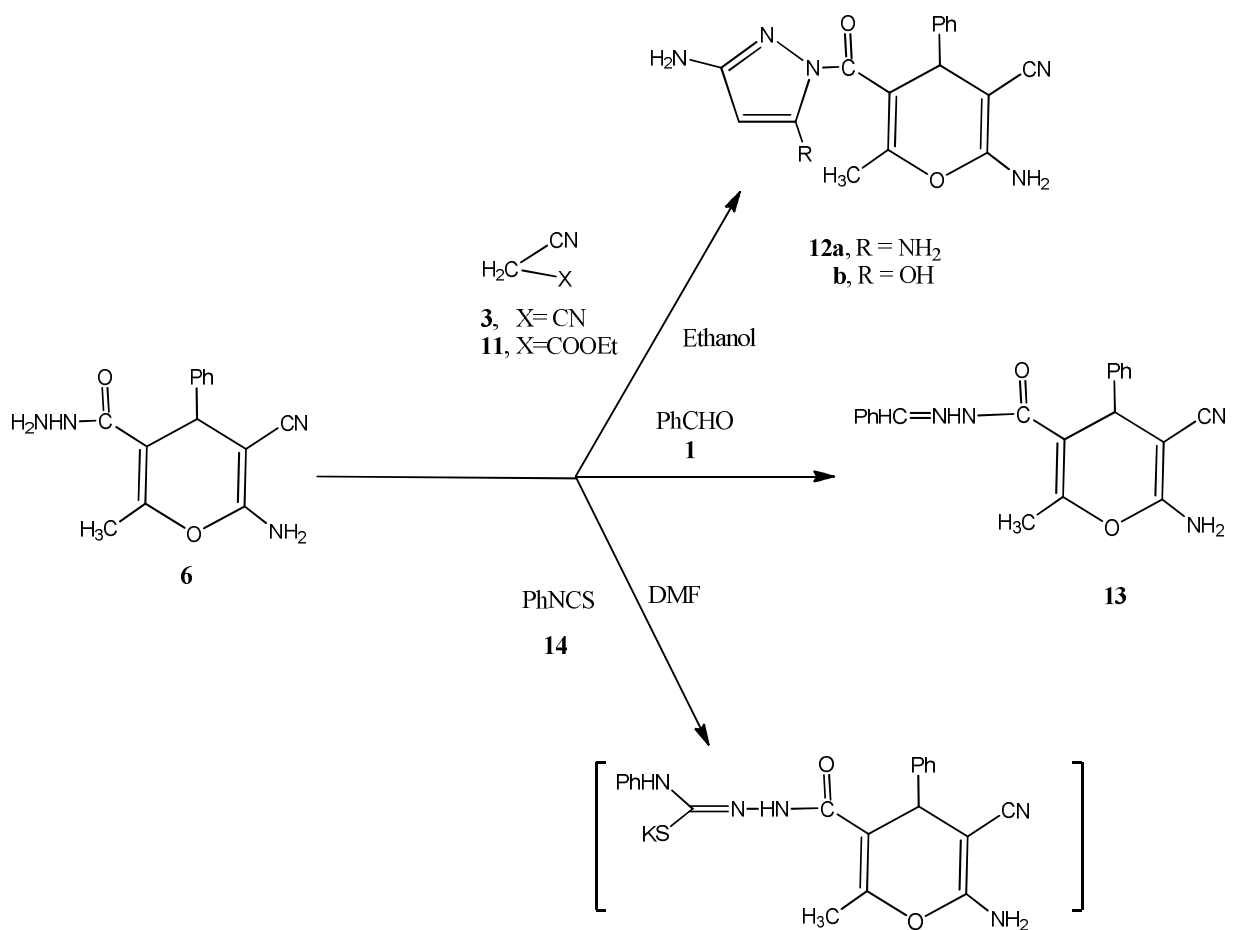

15

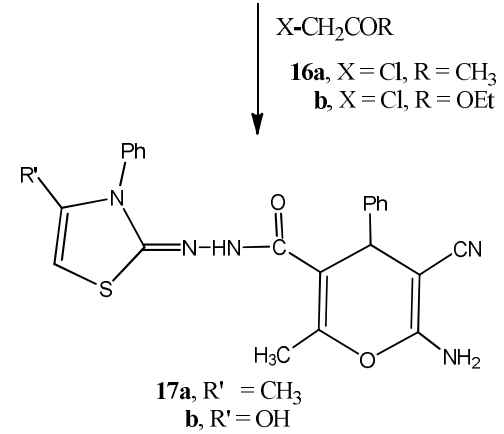

Scheme 2. Synthesis of compounds 12a,b, 15; and $\mathbf{1 7 a}, \mathbf{a}$.

latter compounds were confirmed on the basis of their respective analytical and spectral data. Thus, the ${ }^{1} \mathrm{H}$ NMR of compound 8a showed the presence of two multiplets at $\delta 1.69-1.85$ and 2.49-2.63 ppm equivalent to the four $\mathrm{CH}_{2}$ groups, a singlet at $\delta 4.93 \mathrm{ppm}$ corresponding to the $\mathrm{NH}_{2}$ group, a singlet at $\delta 5.09 \mathrm{ppm}$ indicating the pyran $\mathrm{H}-4$, a multiplet at $\delta 7.26-7.45 \mathrm{ppm}$ for the $\mathrm{C}_{6} \mathrm{H}_{5}$ group and a singlet $\left(\mathrm{D}_{2} \mathrm{O}\right.$ exchangeable for the $\mathrm{NH}$ group. In addition, the ${ }^{13} \mathrm{C} \mathrm{NMR}$ spectrum showed the presence of four signals at $\delta 23.2,28.2,30.5,31.6$ according to the $4 \mathrm{CH}_{2}$ groups, a signal at $\delta 36.6$ equivalent to the $\mathrm{CH}_{3}$ group, a signal at $\delta 51.1$ proving the pyran $\mathrm{C}-4$, a signal at $\delta 116.7$ for the $\mathrm{CN}$ group, four signals at $\delta 120.5,121.8,122.5,124.6$ equivalent to the 
$\mathrm{C}_{6} \mathrm{H}_{5}$ group, four signals at $\delta 132.1,133.2,134.9,138.6$ indicating the pyran $\mathrm{C}$ and two signals at $\delta 164.6,166.4$ corresponding to the $\mathrm{C}=\mathrm{N}$ and $\mathrm{C}=\mathrm{O}$ groups, respectively.

Compound 6 reacted with either ethyl acetoacetate (2) or acetylacetone (9) to give the pyrazole derivatives $\mathbf{1 0 a}$ and $\mathbf{1 0 b}$, respectively (Scheme 1).

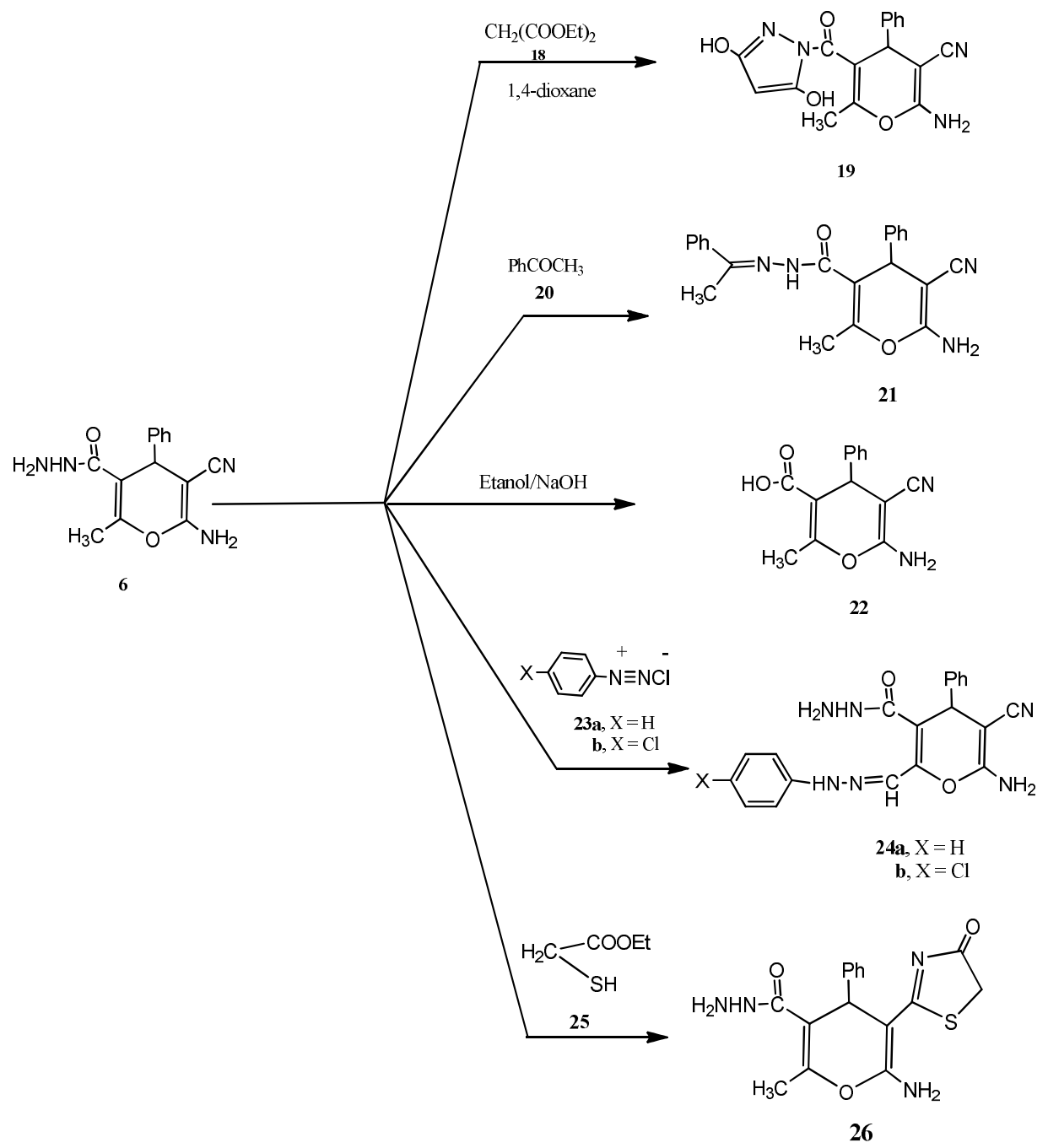

Scheme 3. Synthesis of compounds 19, 21, 22, 24a,b and 26.

Moreover, the reaction of compound $\mathbf{6}$ with either malononitrile (3) or ethyl cyanoacetate (11) afforded the pyrazole derivatives 12a and $\mathbf{1 2} \mathbf{b}$, respectively. The reaction of compound (6) with benzaldehyde (1) gave pyrazole derivatives 13 [26].

Our research group was involved through a comprehensive program involving the reaction of hydrazides and active methylene reagents with phenylisothiocyanate in dimethylformamide solution containing potassium hydroxide to give the intermediate potassium sulphide salt. The 
latter underwent a series of heterocyclization reaction upon reaction with $\alpha$-halocarbonyl compounds to produce thiophene or thiazole derivatives [27-29]. In continuation of this program the reaction of compound 6 with phenylisothiocyanate (14) in dimethylformamide containing potassium hydroxide gave the intermediate potassium sulphide salt $\mathbf{1 5}$. The latter reacted with either chloroacetone or ethyl chloroacetate to give the thiazole derivatives $\mathbf{1 7} \mathbf{a}$ and $\mathbf{1 7} \mathbf{b}$, respectively (Scheme 2).

In addition, the reaction of compound $\mathbf{6}$ with diethylmalonate gave the pyrazole derivative $\mathbf{1 9}$. The reaction of compound $\mathbf{6}$ with acetophenone (20) gave the hydrazide-hydrazone derivative 21. Compound 6 underwent hydrolysis when reacted with ethanolic sodium hydroxide to give the pyran-3-carboxylic acid $\mathbf{2 2}$. The methyl group present in compound $\mathbf{6}$ showed interesting activity toward hydrazone formation, this is attributed to the presence of the $\mathrm{CH}_{3}$ group in ortho position to electronegative COOEt group. Thus, compound $\mathbf{6}$ reacted with either benzenediazonium chloride 23a,b or 4-chlorobenzenediazonium chloride to give the corresponding arylhydrazone derivatives 24a and $\mathbf{2 4 b}$, respectively. Finally, the reaction of compound $\mathbf{6}$ with thioglycollic acid (25) in ethanol containing triethylamine gave the thiazole derivative 26 (Scheme 3).

\section{Cell proliferation assay}

The anti-proliferative activities of the newly synthesized compounds (Table 3) were evaluated against the six cancer cell lines A549, HT-29, MKN-45, U87MG, and SMMC-7721 and H460 using the standard MTT assay in vitro, with foretinib as the positive control [30-32].

The mean values of three independent experiments, expressed as $\mathrm{IC}_{50}$ values, were presented in Table III. Most of the synthesized compounds exhibited potent anti-proliferative activity with $\mathrm{IC}_{50}$ values less than $30 \mu \mathrm{M}$. Generally, the variations of substituents' within the heterocyclic moiety together with the nature of the heterocyclic ring being attached have a notable influence on the anti-proliferative activity.

Table 1. In vitro growth inhibitory effects $\mathrm{IC}_{50}(\mu \mathrm{M})$ of the selected compounds against cancer cell lines \pm SEM.

\begin{tabular}{|c|c|c|c|c|c|c|}
\hline \multirow{2}{*}{ Compound No } & \multicolumn{7}{|c|}{ IC $50 \pm$ SEM $(\mu \mathrm{M})$} \\
\cline { 2 - 7 } & A549 & H460 & HT29 & MKN-45 & U87MG & SMMC-7721 \\
\hline $\mathbf{8 a}$ & $2.37 \pm 0.39$ & $4.58 \pm 1.25$ & $2.31 \pm 1.49$ & $5.62 \pm 1.48$ & $6.39 \pm 1.42$ & $2.87 \pm 1.22$ \\
\hline $\mathbf{8 b}$ & $0.23 \pm 0.15$ & $0.89 \pm 0.41$ & $0.38 \pm 0.17$ & $0.26 \pm 0.12$ & $1.22 \pm 0.65$ & $0.73 \pm 0.22$ \\
\hline $\mathbf{1 0 a}$ & $5.29 \pm 1.48$ & $5.36 \pm 2.32$ & $3.41 \pm 1.23$ & $1.53 \pm 0.92$ & $3.26 \pm 1.06$ & $4.53 \pm 1.33$ \\
\hline $\mathbf{1 0 b}$ & $1.25 \pm 0.63$ & $0.72 \pm 0.28$ & $0.35 \pm 0.28$ & $0.53 \pm 0.26$ & $0.65 \pm 0.42$ & $0.18 \pm 0.05$ \\
\hline $\mathbf{1 1 a}$ & $1.29 \pm 0.74$ & $1.42 \pm 0.47$ & $1.28 \pm 0.63$ & $2.28 \pm 1.15$ & $1.63 \pm 0.46$ & $2.98 \pm 1.63$ \\
\hline $\mathbf{1 2 b}$ & $6.82 \pm 1.53$ & $4.53 \pm 2.15$ & $5.24 \pm 2.31$ & $4.22 \pm 2.08$ & $2.35 \pm 2.31$ & $4.28 \pm 1.29$ \\
\hline $\mathbf{1 3}$ & $6.45 \pm 2.58$ & $8.25 \pm 2.61$ & $6.53 \pm 2.49$ & $6.77 \pm 1.27$ & $4.19 \pm 2.08$ & $6.40 \pm 3.30$ \\
\hline $\mathbf{1 6}$ & $4.63 \pm 1.82$ & $2.93 \pm 1.64$ & $5.28 \pm 1.63$ & $6.48 \pm 1.73$ & $5.27 \pm 1.43$ & $2.29 \pm 0.59$ \\
\hline $\mathbf{1 7 a}$ & $0.61 \pm 0.28$ & $0.58 \pm 0.29$ & $0.48 \pm 0.26$ & $0.62 \pm 0.35$ & $0.52 \pm 0.26$ & $1.59 \pm 0.26$ \\
\hline $\mathbf{1 7 b}$ & $4.53 \pm 1.28$ & $6.70 \pm 1.83$ & $6.27 \pm 2.31$ & $5.83 \pm 1.43$ & $5.28 \pm 2.34$ & $2.34 \pm 2.26$ \\
\hline $\mathbf{1 9}$ & $6.08 \pm 1.28$ & $5.61 \pm 1.72$ & $8.08 \pm 2.27$ & $1.68 \pm 0.49$ & $4.22 \pm 1.53$ & $6.45 \pm 2.14$ \\
\hline $\mathbf{2 1}$ & $0.28 \pm 0.18$ & $0.64 \pm 0.25$ & $0.53 \pm 0.29$ & $0.31 \pm 0.18$ & $0.62 \pm 0.26$ & $0.58 \pm 0.26$ \\
\hline $\mathbf{2 2}$ & $4.26 \pm 1.43$ & $4.42 \pm 2.39$ & $3.42 \pm 1.57$ & $4.52 \pm 1.59$ & $2.42 \pm 0.93$ & $3.38 \pm 0.46$ \\
\hline $\mathbf{2 4 a}$ & $0.34 \pm 0.18$ & $0.37 \pm 0.25$ & $0.52 \pm 0.14$ & $0.33 \pm 0.18$ & $0.92 \pm 0.32$ & $0.83 \pm 0.26$ \\
\hline $\mathbf{2 4 b}$ & $4.24 \pm 3.18$ & $4.25 \pm 2.43$ & $6.52 \pm 2.32$ & $7.48 \pm 2.62$ & $8.58 \pm 2.73$ & $5.82 \pm 2.23$ \\
\hline $\mathbf{2 6}$ & $6.27 \pm 2.12$ & $5.42 \pm 2.38$ & $4.36 \pm 2.59$ & $5.75 \pm 1.48$ & $6.20 \pm 2.38$ & $3.68 \pm 1.42$ \\
\hline Foretinib & $0.08 \pm 0.01$ & $0.18 \pm 0.03$ & $0.15 \pm 0.023$ & $0.03 \pm 0.0055$ & $0.90 \pm 0.13$ & $0.44 \pm 0.062$ \\
\hline
\end{tabular}

Structure activity relationship

It is clear from Table 1 that compounds $8 \mathbf{b}, \mathbf{1 0 b}, \mathbf{1 7 a}, 21$ and $\mathbf{2 4 a}$ were the most active compounds among the tested compounds. Considering the hydrazide-hydrazone derivatives $\mathbf{8 a}$ and $\mathbf{8 b}$, it is 
clear that compound $\mathbf{8 b}$ with the cyclohexylidene moiety showed more inhibitions than compound 8a with the cyclopentylidene moiety. Considering the pyrazole derivatives 10a and 10b, surprisingly compound $10 \mathbf{b}\left(\mathrm{R}=\mathrm{CH}_{3}\right)$ exhibited more inhibitions than 10a $(\mathrm{R}=\mathrm{OH})$ although the latter contains the electronegative $\mathrm{OH}$ group. Similarly, compound 12b exhibited low inhibitions against the six cancer cell lines although it has $\mathrm{OH}$ group within its structure. On the other hand, the pyran derivatives $\mathbf{1 3}$ and $\mathbf{1 5}$ showed low inhibitions against the six cancer cell lines. On the other hand, for the pyran derivatives $17 \mathbf{a}$ and $\mathbf{1 7} \mathbf{b}$, it is obvious that compound $\mathbf{1 7} \mathbf{a}$ $\left(\mathrm{R}=\mathrm{CH}_{3}\right)$ exhibited higher inhibitions than $\mathbf{1 7} \mathbf{b}(\mathrm{R}=\mathrm{OH})$. It is clear from Table 1 that the pyran derivative $\mathbf{2 1}$ has higher inhibitions than the pyran derivatives $\mathbf{1 9}$ and $\mathbf{2 2}$. The high inhibitions of 21 was attributed to the presence of the hydrazide-hydrazone moiety within its structure. For the pyran derivative 22, it was obvious that it had moderate inhibitions toward the cancer cell lines, in addition its highest inhibition was toward the U87MG cell line with $\mathrm{IC}_{50} 2.42 \mu \mathrm{M}$. Considering the arylhydrazone derivatives $\mathbf{2 4 a}$ and $\mathbf{2 4 b}$, it was clear that compound 24a $(X=H)$ exhibited higher inhibitions that $\mathbf{2 4 b}(\mathrm{X}=\mathrm{Cl})$. Finally, the thiazole derivative $\mathbf{2 6}$ exhibited from low to moderate inhibitions against the six cancer cell lines. From the demonstration of such activities of the compounds, it is clear that in most cases the presences of certain heterocyclic and/or certain moiety was the most controlling factor through the high inhibition of the compounds. For example, for compounds $\mathbf{8 a}, \mathbf{b}$ and $\mathbf{2 1}$ the high inhibition was attributed to the presence of the hydrazide-hydrazone moiety. On the other hand, for compounds $\mathbf{1 7} \mathbf{a}$ and $\mathbf{1 7 \mathbf { b }}$ the presence of thiazole ring in their structure was responsible for their activities. For compounds $\mathbf{2 4 a}$ and $\mathbf{2 4 b}$, the presence of the hydrazide group at C-5 and the hydrazone group at C- 6 of the pyran ring increase the inhibitions of such compounds.

\section{Inhibition of selected compounds against tyrosine kinases}

The most potent compounds toward the cancer cell lines compounds $8 \mathbf{b}, \mathbf{1 0 b}, \mathbf{1 1 a}, \mathbf{1 7} \mathbf{a}, 21$ and 24a were further investigated towards the five tyrosine kinases c-kit, FIT-3, VEGFR-2, EGFR and PDGFR and the data were expressed through Table 2. It is obvious from the Table that compound 8b showed high inhibitions toward EGFR and PDGFR kinases with $\mathrm{IC}_{50}$ 's 0.78 and $0.64 \mathrm{nM}$, respectively while it showed moderate inhibitions toward c-Kit and Flt-3 kinases with $\mathrm{IC}_{50}$ 's 1.27 and $1.46 \mathrm{nM}$, respectively. On the other hand, compounds $\mathbf{1 0 b}, 21$ and 24 a expressed high inhibitions toward VEGFR-2, EGFR and PDGFR kinases and moderate inhibitions toward c-Kit and Flt-3 kinases. Interestingly, compounds 11a, 17a showed high inhibitions toward the five tyrosine kinases.

Table 2. Inhibition of tyrosine kinases (Enzyme $\mathrm{IC}_{50}(\mathrm{nM})$ for compounds $\mathbf{8 b}, \mathbf{1 0 b}, \mathbf{1 1 a}, \mathbf{1 7 a}, \mathbf{2 1}$ and $\mathbf{2 4 a}$.

\begin{tabular}{|c|c|c|c|c|c|}
\hline Compound & c-Kit & Flt-3 & VEGFR-2 & EGFR & PDGFR \\
\hline $\mathbf{8 b}$ & 1.27 & 1.46 & 1.38 & 0.78 & 0.64 \\
\hline $\mathbf{1 0 b}$ & 1.28 & 1.21 & 0.93 & 0.28 & 0.70 \\
\hline $\mathbf{1 1 a}$ & 0.27 & 0.28 & 0.31 & 0.58 & 0.44 \\
\hline $\mathbf{1 7 a}$ & 0.23 & 1.06 & 0.68 & 0.48 & 0.49 \\
\hline $\mathbf{2 1}$ & 2.52 & 2.47 & 1.79 & 1.32 & 0.93 \\
\hline $\mathbf{2 4 a}$ & 1.24 & 1.28 & 0.87 & 0.72 & 0.49 \\
\hline Foretinib & 0.19 & 0.17 & 0.20 & 0.13 & 0.26 \\
\hline
\end{tabular}

Inhibition of Pim-1 kinase for compounds 10b,13b,18b,18a and 20

Compounds 8b, 10a, 11a and 17a were selected to examine their Pim-1 kinase inhibition activity (Table 3) as these compounds showed high inhibition towards the tested cancer cell lines at a range of 10 concentrations and the $\mathrm{IC}_{50}$ values were calculated. Compounds 10a, 11a and 17a were the most potent to inhibit Pim-1 kinase with $\mathrm{IC}_{50}$ value of $0.32,0.49$ and 0.28 and $\mu \mathrm{M}$, 
respectively. On the other hand, compounds $\mathbf{8 b}$ was less effective $\left(\mathrm{IC}_{50}>10 \mu \mathrm{M}\right)$. These profiles in combination with cell growth inhibition data of compounds $8 \mathbf{b}, 10 \mathbf{a}, 11 \mathbf{a}$ and $17 \mathbf{a}$ were listed in Table 3 and indicated that Pim-1 was a potential target of these compounds where SGI-1776 was used as positive control with $\mathrm{IC}_{50} 0.048 \mu \mathrm{M}$ in the assay.

Table 3. Inhibition of Pim-1 kinase for compounds $8 \mathbf{b}, \mathbf{1 0 a}, 11 \mathbf{a}$ and 17a

\begin{tabular}{|c|c|c|}
\hline Compound & Inhibition ratio At $10 \mu \mathrm{M}$ & $\mathrm{IC}_{50}(\mu \mathrm{M})$ \\
\hline $\mathbf{8 b}$ & 38 & $>100$ \\
\hline $\mathbf{1 0 a}$ & 94 & 0.32 \\
\hline $\mathbf{1 1 a}$ & 90 & 0.49 \\
\hline $\mathbf{1 7 a}$ & 96 & 0.28 \\
\hline SGI-1776 & - & 0.048 \\
\hline
\end{tabular}

It is clear from Table 3 that compounds 10a, 11a and 17a are of the highest inhibitions toward Pim-1 kinase with $\mathrm{IC}_{50}$ 's $0.32,0.49$ and $0.28 \mu \mathrm{M}$, respectively.

\section{EXPERIMENTAL}

\section{Chemistry}

Through this work the melting points of the synthesized compounds were recorded on Buchi melting point apparatus D-545. The IR spectra ( $\mathrm{KBr}$ discs) were measured on Bruker Vector 22 instrument. ${ }^{13} \mathrm{C}$ NMR and ${ }^{1} \mathrm{H}$ NMR spectra were measured on Bruker DPX300 instrument in DMSO- $d_{6}$ with TMS as internal standard. Mass spectra were measured using EIMS (Shimadzu) and ESI-esquire 3000 Bruker Daltonics instrument. Elemental analyses were measured using the Micro-analytical Data center at Cairo University. All reactions was monitored by TLC on $2 \times 5$ $\mathrm{cm}$ pre-coated silica gel $60 \mathrm{~F} 254$ plates of thickness of $0.25 \mathrm{~mm}$ (Merck) for getting complete reactions.

Ethyl 6-amino-5-cyano-2-methyl-4-phenyl-4H-pyran-3-carboxylate (4). A mixture of benzaldehyde $(1.06 \mathrm{~g}, 0.01 \mathrm{~mol})$, ethyl acetoacetate $(1.30 \mathrm{~g}, 0.01 \mathrm{~mol})$ and malononitrile $(0.66 \mathrm{~g}$, $0.01 \mathrm{~mol})$ in absolute ethanol $(60 \mathrm{~mL})$ containing triethylamine $(1.0 \mathrm{~mL})$ was heated under reflux for $3 \mathrm{~h}$ then was left to cool. The solid product produced upon cooling was collected by filtration.

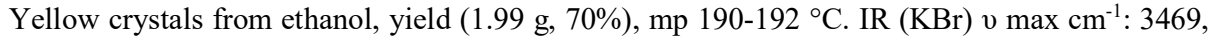
$3342\left(\mathrm{NH}_{2}\right), 3055\left(\mathrm{CH}\right.$, aromatic), 2920, $2867\left(\mathrm{CH}_{2}, \mathrm{CH}_{3}\right), 2220(\mathrm{CN}), 1703(\mathrm{C}=\mathrm{O}), 1630(\mathrm{C}=\mathrm{C})$; ${ }^{1} \mathrm{H}$ NMR (DMSO- $\left.d_{6}, 300 \mathrm{MHz}\right): \delta=1.13\left(\mathrm{t}, 3 \mathrm{H}, J=7.26 \mathrm{~Hz}, \mathrm{OCH}_{2} \underline{\mathrm{CH}}_{3}\right), 2.70\left(\mathrm{~s}, 3 \mathrm{H}, \mathrm{CH}_{3}\right)$, $4.22\left(\mathrm{q}, 2 \mathrm{H}, J=7.26 \mathrm{~Hz}, \mathrm{OCH}_{2} \mathrm{CH}_{3}\right), 4.92\left(\mathrm{~s}, 2 \mathrm{H}, \mathrm{D}_{2} \mathrm{O}\right.$ exchangeable, $\left.\mathrm{NH}_{2}\right), 5.08(\mathrm{~s}, 1 \mathrm{H}$, pyran $\mathrm{H}-4), 7.23-7.55\left(\mathrm{~m}, 5 \mathrm{H}, \mathrm{C}_{6} \mathrm{H}_{5}\right) ;{ }^{13} \mathrm{C} \mathrm{NMR}$ (DMSO- $\left.d_{6}, 75 \mathrm{MHz}\right): \delta 16.8\left(\mathrm{OCH}_{2} \mathrm{CH}_{3}\right), 36.8\left(\mathrm{CH}_{3}\right)$, $50.2\left(\mathrm{OCH}_{2} \mathrm{CH}_{3}\right), 51.0$ (pyran C-4), $116.8(\mathrm{CN}), 120.4,121.8,124.6,126.4\left(\mathrm{C}_{6} \mathrm{H}_{5}\right), 132.6,134.2$, 136.0, 138.4 (pyran C), $164.8(\mathrm{C}=\mathrm{O})$. Anal. calcd. for $\mathrm{C}_{16} \mathrm{H}_{16} \mathrm{~N}_{2} \mathrm{O}_{3}(284.31)$ : $\mathrm{C}, 67.59 ; \mathrm{H}, 5.67 ; \mathrm{N}$, $9.85 \%$. Found: C, 67.36; H, 5.73; N, 10.02\%. MS: m/z $284\left[\mathrm{M}^{+}, 65 \%\right]$.

6-Amino-5-cyano-2-methyl-4-phenyl-4H-pyran-3-carbohydrazide (6). To a solution of compound $4(2.84 \mathrm{~g}, 0.01 \mathrm{~mol})$ in 1,4-dioxane $(40 \mathrm{~mL})$ hydrazine hydrate was added. The reaction mixture was heated under reflux for $4 \mathrm{~h}$ then was poured onto ice/water mixture containing a few drops of hydrochloric acid and the formed solid product was collected by filtration. Yellow crystals from ethanol, yield $(2.02 \mathrm{~g}, 75 \%), \mathrm{Mp} 143-145^{\circ} \mathrm{C}$. IR $(\mathrm{KBr}) v \max \mathrm{cm}^{-1}: 3478,3351\left(\mathrm{NH}_{2}\right), 3055(\mathrm{CH}$, aromatic), 2920, $2867\left(\mathrm{CH}_{2}, \mathrm{CH}_{3}\right), 2220(\mathrm{CN}), 1689(\mathrm{C}=\mathrm{O}), 1630(\mathrm{C}=\mathrm{C}) ;{ }^{1} \mathrm{H}$ NMR (DMSO- $d_{6}$, $300 \mathrm{MHz}): \delta=2.73\left(\mathrm{~s}, 3 \mathrm{H}, \mathrm{CH}_{3}\right), 4.96,5.21\left(2 \mathrm{~s}, 4 \mathrm{H}, \mathrm{D}_{2} \mathrm{O}\right.$ exchangeable, $\left.2 \mathrm{NH}_{2}\right), 5.10(\mathrm{~s}, 1 \mathrm{H}$, pyran $\mathrm{H}-4), 7.24-7.48\left(\mathrm{~m}, 5 \mathrm{H}, \mathrm{C}_{6} \mathrm{H}_{5}\right), 8.28\left(\mathrm{~s}, 1 \mathrm{H}, \mathrm{D}_{2} \mathrm{O}\right.$ exchangeable, $\left.\mathrm{NH}\right) ;{ }^{13} \mathrm{C}$ NMR (DMSO- 
$\left.d_{6}, 75 \mathrm{MHz}\right): \delta 36.4\left(\mathrm{CH}_{3}\right), 51.3$ (pyran C-4), $116.8(\mathrm{CN}), \quad 120.2,122.4,123.9,125.8\left(\mathrm{C}_{6} \mathrm{H}_{5}\right)$, 132.3, 133.8, 135.6, 138.2 (pyran $\mathrm{C}$ ), $163.8(\mathrm{C}=\mathrm{O})$. Anal. calcd. for $\mathrm{C}_{14} \mathrm{H}_{14} \mathrm{~N}_{4} \mathrm{O}_{2}$ (270.29): $\mathrm{C}$, 62.21 ; H, 5.22; N, 20.73 \%. Found: C, 62.08; H, 5.41; N, 20.59\%. MS: m/z 270 [M+ , 80\%].

General procedure for the synthesis of the hydrazide-hydrazone derivatives $\boldsymbol{8 a}, \boldsymbol{b}$. To a solution of compound $6(2.70 \mathrm{~g}, 0.01 \mathrm{~mol})$ in 1,4-dioxane $(40 \mathrm{~mL})$ either cyclopentanone $(0.84 \mathrm{~g}, 0.01 \mathrm{~mol})$ or cyclohexanone $(0.98 \mathrm{~g}, 0.01 \mathrm{~mol})$ was added. The reaction mixture was heated under reflux for $2 \mathrm{~h}$ then was left to cool and the formed solid product was collected by filtration.

6-Amino-5-cyano- $N^{\prime}$-cyclopentylidene-2-methyl-4-phenyl-4H-pyran-3-carbohydrazide (8a). Brown crystals from ethanol, yield $(2.28 \mathrm{~g}, 68 \%), \mathrm{mp} 70-75^{\circ} \mathrm{C}$. IR $(\mathrm{KBr}) v \max \mathrm{cm}^{-1}: 3461-3339$ $\left(\mathrm{NH}, \mathrm{NH}_{2}\right), 3055\left(\mathrm{CH}\right.$, aromatic), 2928, $2869\left(\mathrm{CH}_{2}, \mathrm{CH}_{3}\right), 2220(\mathrm{CN}), 1688(\mathrm{C}=\mathrm{O}), 1630(\mathrm{C}=\mathrm{C})$; ${ }^{1} \mathrm{H}$ NMR (DMSO- $\left.d_{6}, 300 \mathrm{MHz}\right): \delta=1.69-1.85\left(\mathrm{~m}, 4 \mathrm{H}, 2 \mathrm{CH}_{2}\right), 2.49-2.63\left(\mathrm{~m}, 4 \mathrm{H}, 2 \mathrm{CH}_{2}\right), 2.76(\mathrm{~s}$, $\left.3 \mathrm{H}, \mathrm{CH}_{3}\right), 4.93\left(\mathrm{~s}, 2 \mathrm{H}, \mathrm{D}_{2} \mathrm{O}\right.$ exchangeable, $\left.\mathrm{NH}_{2}\right), 5.09(\mathrm{~s}, 1 \mathrm{H}$, pyran $\mathrm{H}-4), 7.26-7.45\left(\mathrm{~m}, 5 \mathrm{H}, \mathrm{C}_{6} \mathrm{H}_{5}\right)$, $8.32\left(\mathrm{~s}, 1 \mathrm{H}, \mathrm{D}_{2} \mathrm{O}\right.$ exchangeable, NH); ${ }^{13} \mathrm{C}$ NMR (DMSO- $\left.d_{6}, 75 \mathrm{MHz}\right): \delta 23.2,28.2,30.5,31.6$ $\left(4 \mathrm{CH}_{2}\right), 36.6\left(\mathrm{CH}_{3}\right), 51.1$ (pyran C-4), $116.7(\mathrm{CN}), 120.5,121.8,122.5,124.6\left(\mathrm{C}_{6} \mathrm{H}_{5}\right), 132.1$, 133.2, 134.9, 138.6 (pyran C), $164.6(\mathrm{C}=\mathrm{N}), 166.4(\mathrm{CO})$. Anal. calcd. for $\mathrm{C}_{19} \mathrm{H}_{20} \mathrm{~N}_{4} \mathrm{O}_{2}(336.39)$ : C, 67.84; H, 5.99; N, $16.66 \%$. Found: C, 68.02; H, 5.83; N, 16.80\%. MS: m/z 336 [M $\mathrm{M}^{+}, 68 \%$ ].

6-Amino-5-cyano- $N$ '-cyclohexylidene-2-methyl-4-phenyl-4H-pyran-3-carbohydrazide $\quad(\mathbf{8 b})$. Browm crystals from ethanol, yield $(2.59 \mathrm{~g}, 74 \%), \mathrm{mp} 100{ }^{\circ} \mathrm{C}$. IR $(\mathrm{KBr}) v \operatorname{max~cm}{ }^{-1}: 3473-3362$ $\left(\mathrm{NH}, \mathrm{NH}_{2}\right), 3055\left(\mathrm{CH}\right.$, aromatic), 2952, $2841\left(\mathrm{CH}_{2}, \mathrm{CH}_{3}\right), 2220(\mathrm{CN}), 1702(\mathrm{C}=\mathrm{O}), 1632(\mathrm{C}=\mathrm{C})$; ${ }^{1} \mathrm{H}$ NMR (DMSO- $\left.d_{6}, 300 \mathrm{MHz}\right): \delta=1.49-1.87\left(\mathrm{~m}, 6 \mathrm{H}, 3 \mathrm{CH}_{2}\right), 2.32-2.72\left(\mathrm{~m}, 4 \mathrm{H}, 2 \mathrm{CH}_{2}\right), 2.78(\mathrm{~s}$, $\left.3 \mathrm{H}, \mathrm{CH}_{3}\right), 4.96\left(\mathrm{~s}, 2 \mathrm{H}, \mathrm{D}_{2} \mathrm{O}\right.$ exchangeable, $\left.\mathrm{NH}_{2}\right), 5.12(\mathrm{~s}, 1 \mathrm{H}$, pyran $\mathrm{H}-4), 7.25-7.49\left(\mathrm{~m}, 5 \mathrm{H}, \mathrm{C}_{6} \mathrm{H}_{5}\right)$, 8.30 (s, $1 \mathrm{H}, \mathrm{D}_{2} \mathrm{O}$ exchangeable, NH); ${ }^{13} \mathrm{C}$ NMR (DMSO- $\left.d_{6}, 75 \mathrm{MHz}\right): \delta 24.6,26.8,28.5,30.8$, $32.4\left(5 \mathrm{CH}_{2}\right), 36.4\left(\mathrm{CH}_{3}\right), 51.3$ (pyran C-4), $116.9(\mathrm{CN}), 120.2,122.6,123.8,125.0\left(\mathrm{C}_{6} \mathrm{H}_{5}\right), 132.3$, 133.5, 134.6, 138.2 (pyran C), $164.9(\mathrm{C}=\mathrm{N}), 168.3(\mathrm{CO})$. Anal. calcd. for $\mathrm{C}_{20} \mathrm{H}_{22} \mathrm{~N}_{4} \mathrm{O}_{2}(350.41)$ : C, 68.55; H, 6.33; N, 15.99\%. Found: C, 68.43; H, 6.25; N, 16.25\%. MS: m/z 350 [M+ , 45\%].

General procedure for the synthesis of the 1H-pyrazole-1-carbonyl)-6-methyl-4-phenyl-4H-pyran derivatives $\mathbf{1 0 a}, \boldsymbol{b}$. Either of ethyl acetoacetate $(1.30 \mathrm{~mL}, 0.01 \mathrm{~mol})$ or acetylacetone $(1.00 \mathrm{~mL}$, $0.01 \mathrm{~mol})$ in absolute ethanol $(50 \mathrm{~mL})$ was added to a solution of compound $6(2.70 \mathrm{~g}, 0.01 \mathrm{~mol})$ in absolute ethanol $(40 \mathrm{~mL})$. The reaction mixture, in each case, was heated under reflux for $2 \mathrm{~h}$ then poured onto ice/water mixture and the formed solid product was collected by filtration.

2-Amino-5-(5-hydroxy-3-methyl-1H-pyrazole-1-carbonyl)-6-methyl-4-phenyl-4H-pyran-3-carbonitrile (10a). Brown crystals from ethanol, yield $(2.21 \mathrm{~g}, 66 \%), \mathrm{mp} 80^{\circ} \mathrm{C}$. IR $(\mathrm{KBr}) v \operatorname{max~cm}^{-1}$ : 3529, $3328\left(\mathrm{OH}, \mathrm{NH}_{2}\right), 3055\left(\mathrm{CH}\right.$, aromatic), 2928, $2863\left(\mathrm{CH}_{2}, \mathrm{CH}_{3}\right), 2220(\mathrm{CN}), 1688(\mathrm{C}=\mathrm{O})$, $1630(\mathrm{C}=\mathrm{C}) ;{ }^{1} \mathrm{H}$ NMR (DMSO- $\left.d_{6}, 300 \mathrm{MHz}\right): \delta=2.78,2.84\left(2 \mathrm{~s}, 6 \mathrm{H}, 2 \mathrm{CH}_{3}\right), 4.94\left(\mathrm{~s}, 2 \mathrm{H}, \mathrm{D}_{2} \mathrm{O}\right.$ exchangeable, $\left.\mathrm{NH}_{2}\right), 5.08,6.11(2 \mathrm{~s}, 2 \mathrm{H}$, pyran $\mathrm{H}-4), 7.26-7.46\left(\mathrm{~m}, 5 \mathrm{H}, \mathrm{C}_{6} \mathrm{H}_{5}\right), 10.26\left(\mathrm{~s}, 1 \mathrm{H}, \mathrm{D}_{2} \mathrm{O}\right.$ exchangeable, $\mathrm{OH}) ;{ }^{13} \mathrm{C}$ NMR (DMSO- $\left.d_{6}, 75 \mathrm{MHz}\right): \delta 36.2,37.8\left(2 \mathrm{CH}_{3}\right), 51.4$ (pyran C-4), 116.6 $(\mathrm{CN}), 120.4,121.8,124.6,125.2\left(\mathrm{C}_{6} \mathrm{H}_{5}\right), 132.0,133.2,134.8,135.4,137.6,138.1$ (pyran, pyrazole C), $163.9(\mathrm{C}=\mathrm{N}), 166.2(\mathrm{C}=\mathrm{O})$. Anal. calcd. for $\mathrm{C}_{18} \mathrm{H}_{16} \mathrm{~N}_{4} \mathrm{O}_{3}(336.34)$ : C, 64.28; H, 4.79; N, 16.66\%. Found: C, 64.35; H, 4.93; N, 16.80\%. MS: m/z 336 [M+, 54\%].

2-Amino-5-(3,5-dimethyl-1H-pyrazole-1-carbonyl)-6-methyl-4-phenyl-4H-pyran-3-carbonitrile (10b). Deep yellow crystals from ethanol, yield $(2.33 \mathrm{~g}, 70 \%), \mathrm{mp} 90-92^{\circ} \mathrm{C}$. IR $(\mathrm{KBr})$ v $\operatorname{max~cm}{ }^{-}$ ${ }^{1}:$ 3479, $3342\left(\mathrm{NH}_{2}\right), 3055\left(\mathrm{CH}\right.$, aromatic), 2960, $2883\left(\mathrm{CH}_{2}, \mathrm{CH}_{3}\right), 2222(\mathrm{CN}), 1689(\mathrm{C}=\mathrm{O}), 1632$ $(\mathrm{C}=\mathrm{C}) ;{ }^{1} \mathrm{H}$ NMR $\left(\mathrm{DMSO}-d_{6}, 300 \mathrm{MHz}\right): \delta=2.74,2,80,2.82\left(3 \mathrm{~s}, 9 \mathrm{H}, 3 \mathrm{CH}_{3}\right), 4.93\left(\mathrm{~s}, 2 \mathrm{H}, \mathrm{D}_{2} \mathrm{O}\right.$ exchangeable, $\left.\mathrm{NH}_{2}\right), 5.11,6.16(2 \mathrm{~s}, 2 \mathrm{H}$, pyran $\mathrm{H}-4$ pyrazole $\mathrm{H}-4), 7.23-7.48\left(\mathrm{~m}, 5 \mathrm{H}, \mathrm{C}_{6} \mathrm{H}_{5}\right) ;{ }^{13} \mathrm{C}$ NMR (DMSO- $\left.d_{6}, 75 \mathrm{MHz}\right): \delta 36.1,37.3,38.2\left(3 \mathrm{CH}_{3}\right), 51.2$ (pyran C-4), $116.9(\mathrm{CN}), 120.1$, 
121.3, 123.9, $125.6\left(\mathrm{C}_{6} \mathrm{H}_{5}\right), 132.1,132.9,133.4,134.5,137.2,138.4$ (pyran, pyrazole $\left.\mathrm{C}\right), 164.0$ $(\mathrm{C}=\mathrm{N}), 167.8(\mathrm{C}=\mathrm{O})$. Anal. calcd. for $\mathrm{C}_{19} \mathrm{H}_{18} \mathrm{~N}_{4} \mathrm{O}_{2}(334.37)$ : $\mathrm{C}, 68.25 ; \mathrm{H}, 5.43 ; \mathrm{N}, 16.76 \%$. Found: C, 68.44; H, 5.72; N, 16.58\%. MS: m/z $334\left[\mathrm{M}^{+}, 68 \%\right]$.

General procedure for the synthesis of the pyrazole-1-carbonyl)-6-methyl-4-phenyl-4H-pyran derivatives 12a,b. To a solution of compound $6(2.70 \mathrm{~g}, 0.01 \mathrm{~mol})$ in absolute ethanol $(50 \mathrm{~mL})$ either malononitrile $(0.66 \mathrm{~g}, 0.01 \mathrm{~mol})$ or ethyl cyanoacetate $(1.13 \mathrm{~g}, 0.01 \mathrm{~mol})$ was added. The reaction mixture, in each case, was heated under reflux for $2 \mathrm{~h}$ then poured onto ice/water mixture containing a few drops of hydrochloric acid. The produced solid product, in each case, was collected by filtration.

2-Amino-5-(3,5-diamino-1H-pyrazole-1-carbonyl)-6-methyl-4-phenyl-4H-pyran-3-carbonitrile (12a). Brown crystals from ethanol, yield $(2.55 \mathrm{~g}, 76 \%), \mathrm{mp} 100{ }^{\circ} \mathrm{C}$. IR (KBr) v max cm${ }^{-1}: 3468$, $3362\left(\mathrm{OH}, \mathrm{NH}_{2}\right), 3057\left(\mathrm{CH}\right.$, aromatic), 2980, $2869\left(\mathrm{CH}_{2}, \mathrm{CH}_{3}\right), 2220(\mathrm{CN}), 1689(\mathrm{C}=\mathrm{O}), 1630$ $(\mathrm{C}=\mathrm{C}) ;{ }^{1} \mathrm{H}$ NMR $\left(\mathrm{DMSO}-d_{6}, 300 \mathrm{MHz}\right): \delta=2.76\left(\mathrm{~s}, 3 \mathrm{H}, \mathrm{CH}_{3}\right), 4.90,4.96,5.25\left(3 \mathrm{~s}, 6 \mathrm{H}, \mathrm{D}_{2} \mathrm{O}\right.$ exchangeable, $\left.3 \mathrm{NH}_{2}\right), 5.09,6.14(2 \mathrm{~s}, 2 \mathrm{H}$, pyran $\mathrm{H}-4$ pyrazole $\mathrm{H}-4), 7.24-7.43\left(\mathrm{~m}, 5 \mathrm{H}, \mathrm{C}_{6} \mathrm{H}_{5}\right) ;{ }^{13} \mathrm{C}$ NMR (DMSO- $\left.d_{6}, 75 \mathrm{MHz}\right): \delta 36.8\left(\mathrm{CH}_{3}\right), 51.3$ (pyran C-4), $116.6(\mathrm{CN}), 120.4,121.8,124.6$, $125.2\left(\mathrm{C}_{6} \mathrm{H}_{5}\right), 132.0,133.2,133.2,134.8,137.6,138.0$ (pyran, pyrazole $\left.\mathrm{C}\right), 164.2(\mathrm{C}=\mathrm{N}), 166.2$ $(\mathrm{C}=\mathrm{O})$. Anal. calcd. for $\mathrm{C}_{17} \mathrm{H}_{16} \mathrm{~N}_{6} \mathrm{O}_{2}(336.35)$ : $\mathrm{C}, 60.71 ; \mathrm{H}, 4.79 ; \mathrm{N}, 24.99 \%$. Found: $\mathrm{C}, 60.93 ; \mathrm{H}$, 4.46; N, 25.27\%. MS: m/z $336[\mathrm{M}+, 75 \%]$.

2-Amino-5-(3-amino-5-hydroxy-1H-pyrazole-1-carbonyl)-6-methyl-4-phenyl-4H-pyran-3-carbonitrile (12b). Deep yelow crystals from ethanol, yield $(2.42 \mathrm{~g}, 72 \%), \mathrm{mp} 97-100{ }^{\circ} \mathrm{C}$. IR (KBr) v $\max \mathrm{cm}^{-1}$ : 3574, 3368( $\left.\mathrm{OH}, \mathrm{NH}_{2}\right), 3055\left(\mathrm{CH}\right.$, aromatic), $2982\left(\mathrm{CH}_{3}\right), 2220(\mathrm{CN}), 1689(\mathrm{C}=\mathrm{O})$, $1630(\mathrm{C}=\mathrm{C}) ;{ }^{1} \mathrm{H}$ NMR (DMSO- $\left.d_{6}, 300 \mathrm{MHz}\right): \delta=2.78\left(\mathrm{~s}, 3 \mathrm{H}, \mathrm{CH}_{3}\right), 4.94,5.23\left(2 \mathrm{~s}, 4 \mathrm{H}, \mathrm{D}_{2} \mathrm{O}\right.$ exchangeable, $\left.2 \mathrm{NH}_{2}\right), 5.06,6.12(2 \mathrm{~s}, 2 \mathrm{H}$, pyran $\mathrm{H}-4$ pyrazole $\mathrm{H}-4), 7.22-7.40\left(\mathrm{~m}, 5 \mathrm{H}, \mathrm{C}_{6} \mathrm{H}_{5}\right)$, $10.22\left(\mathrm{~s}, 1 \mathrm{H}, \mathrm{D}_{2} \mathrm{O}\right.$ exchangeable, $\left.\mathrm{OH}\right) ;{ }^{13} \mathrm{C}$ NMR (DMSO- $\left.d_{6}, 75 \mathrm{MHz}\right): \delta 36.4\left(\mathrm{CH}_{3}\right), 51.4$ (pyran C-4), $116.9(\mathrm{CN}), 120.7,122.5,124.1,125.8\left(\mathrm{C}_{6} \mathrm{H}_{5}\right), 132.1,133.6,133.8,134.4,137.2,138.3$ (pyran, pyrazole C), $164.5(\mathrm{C}=\mathrm{N}), 166.8(\mathrm{C}=\mathrm{O})$. Anal. calcd. for $\mathrm{C}_{17} \mathrm{H}_{15} \mathrm{~N}_{5} \mathrm{O}_{3}$ (337.33): $\mathrm{C}, 60.53$; H, 4.48; N, 20.76\%. Found: C, 60.72; H, 4.30; N, 20.51\%. MS: m/z 337 [M+ $\left.\mathrm{M}^{+}, 50 \%\right]$.

6-Amino- $N$-benzylidene-5-cyano-2-methyl-4-phenyl-4H-pyran-3-carbohydrazide (13). To a solution of compound $6(2.70 \mathrm{~g}, 0.01 \mathrm{~mol})$ in 1,4-dioxane $(40 \mathrm{~mL})$ benzaldehyde $(1.08 \mathrm{~g}, 0.01$ mol) was added. The reaction mixture was heated under reflux for $2 \mathrm{~h}$ then was poured onto ice/water containing a few drops of hydrochloric acid and the formed solid product was collected by filtration. Brown crystals from ethanol, yield $(2.43 \mathrm{~g}, 68 \%), \mathrm{mp} 90-93{ }^{\circ} \mathrm{C}$. IR $(\mathrm{KBr}) v \mathrm{max}$ $\mathrm{cm}^{-1}: 3488,3360\left(\mathrm{NH}_{2}\right), 3055\left(\mathrm{CH}\right.$, aromatic), $2920\left(\mathrm{CH}_{3}\right), 2220(\mathrm{CN}), 1688(\mathrm{C}=\mathrm{O}), 1630(\mathrm{C}=\mathrm{C})$; ${ }^{1} \mathrm{H}$ NMR (DMSO- $\left.d_{6}, 300 \mathrm{MHz}\right): \delta=2.71\left(\mathrm{~s}, 3 \mathrm{H}, \mathrm{CH}_{3}\right), 4.96\left(\mathrm{~s}, 2 \mathrm{H}, \mathrm{D}_{2} \mathrm{O}\right.$ exchangeable, $\left.\mathrm{NH}_{2}\right)$, $5.12(\mathrm{~s}, 1 \mathrm{H}$, pyran $\mathrm{H}-4), 6.80(\mathrm{~s}, 1 \mathrm{H}, \mathrm{CH}=\mathrm{N}), 7.25-7.49\left(\mathrm{~m}, 10 \mathrm{H}, 2 \mathrm{C}_{6} \mathrm{H}_{5}\right), 8.28\left(\mathrm{~s}, 1 \mathrm{H}, \mathrm{D}_{2} \mathrm{O}\right.$ exchangeable, NH); ${ }^{13} \mathrm{C}$ NMR (DMSO- $\left.d_{6}, 75 \mathrm{MHz}\right): \delta 36.46\left(\mathrm{CH}_{3}\right), 51.2$ (pyran C-4), 116.8 $(\mathrm{CN}), \quad 120.2,121.4,121.8,122.4,123.9,124.0,1246,125.9\left(\mathrm{C}_{6} \mathrm{H}_{5}\right), 132.2,133.4,136.8,138.9$ (pyran C), $164.4(\mathrm{C}=\mathrm{O})$. Anal. calcd. for $\mathrm{C}_{21} \mathrm{H}_{18} \mathrm{~N}_{4} \mathrm{O}_{2}$ (358.39): C, 70.38; H, 5.06; N, 15.63\%. Found: C, 70.15; H, 5.26; N, 15.42\%. MS: m/z 358 [M+, 65\%].

General procedure for the synthesis of the phenylthiazol-2(3H)-ylidene)-4-phenyl-4H-pyran derivatives $17 \boldsymbol{a}, \boldsymbol{b}$. To a solution of compound $6(2.70 \mathrm{~g}, 0.01 \mathrm{~mol})$ in dimethylformamide $(30 \mathrm{~mL})$ containing potassium hydroxide $(0.80 \mathrm{~g}, 0.02 \mathrm{~mol})$ phenylisothiocyanate $(1.30 \mathrm{~g}, 0.01 \mathrm{~mol})$ was added. The reaction mixture was stirred at room temperature for $24 \mathrm{~h}$ then either chloroacetone $(0.92 \mathrm{~g}, 0.01 \mathrm{~mol})$ or $\alpha$-chloroethyl acetate $(1.67 \mathrm{~g}, 0.01 \mathrm{~mol})$ was added, The whole reaction mixture was stirred at room temperature for another $24 \mathrm{~h}$ then was poured onto ice/water mixture 
containing drops of hydrochloric acid (till $\mathrm{pH} \mathrm{6)}$ ) and the formed solid product, in each case, was collected by filtration.

6-Amino-5-cyano-2-methyl-N'-(4-methyl-3-phenylthiazol-2(3H)-ylidene)-4-phenyl-4H-pyran-3carbohydrazide (17a). Brown crystals from acetic acid, yield $(2.65 \mathrm{~g}, 60 \%)$, mp $160-162{ }^{\circ} \mathrm{C}$. IR $(\mathrm{KBr})$ v max cm${ }^{-1}: 3474,3382\left(\mathrm{NH}, \mathrm{NH}_{2}\right), 3054\left(\mathrm{CH}\right.$, aromatic), $2984\left(\mathrm{CH}_{3}\right), 2222(\mathrm{CN}), 1688$ $(\mathrm{C}=\mathrm{O}), 1630(\mathrm{C}=\mathrm{C}) ;{ }^{1} \mathrm{H}$ NMR (DMSO- $\left.d_{6}, 300 \mathrm{MHz}\right): \delta=2.74,2.86\left(2 \mathrm{~s}, 6 \mathrm{H}, 2 \mathrm{CH}_{3}\right), 4.96(\mathrm{~s}, 2 \mathrm{H}$, $\mathrm{D}_{2} \mathrm{O}$ exchangeable, $\left.\mathrm{NH}_{2}\right), 5.09,6.53(2 \mathrm{~s}, 2 \mathrm{H}$, pyran $\mathrm{H}-4$, thiazole $\mathrm{H}-5), 7.22-7.46(\mathrm{~m}, 10 \mathrm{H}$, $\left.2 \mathrm{C}_{6} \mathrm{H}_{5}\right), 8.26\left(\mathrm{~s}, 1 \mathrm{H}, \mathrm{D}_{2} \mathrm{O}\right.$ exchaneable, $\left.\mathrm{NH}\right) ;{ }^{13} \mathrm{C} \mathrm{NMR}$ (DMSO- $\left.d_{6}, 75 \mathrm{MHz}\right): \delta 36.2,38.5\left(2 \mathrm{CH}_{3}\right)$, 51.1 (pyran C-4), $116.8(\mathrm{CN}), 120.1,121.3,121.9,123.8124 .2,125.6,125.8,126.1\left(2 \mathrm{C}_{6} \mathrm{H}_{5}\right)$, 132.2, 133.5, 133.3, 134.2, 135.0, 137.3 (pyran, thiazole $\mathrm{C}), 166.3(\mathrm{C}=\mathrm{N}), 166.8(\mathrm{C}=\mathrm{O})$. Anal. calcd. for $\mathrm{C}_{24} \mathrm{H}_{21} \mathrm{~N}_{5} \mathrm{O}_{2} \mathrm{~S}$ (443.52): C, 64.99; H, 4.77; N, 15.79; $\mathrm{S}, 7.23 \%$. Found: C, 65.22; H, 4.80; $\mathrm{N}, 15.62 ; \mathrm{S}, 7.08 \%$. MS: $\mathrm{m} / \mathrm{z} 443\left[\mathrm{M}^{+}, 46 \%\right]$.

6-Amino-5-cyano-N'-(4-hydroxy-3-phenylthiazol-2(3H)-ylidene)-2-methyl-4-phenyl-4H-pyran3 -carbohydrazide $(\mathbf{1 7 b})$. Brown crystals from acetic acid, yield $(3.11 \mathrm{~g}, 70 \%), \mathrm{mp} 165{ }^{\circ} \mathrm{C}$. IR $(\mathrm{KBr}) v \max \mathrm{cm}^{-1}: 3582,3359\left(\mathrm{OH}, \mathrm{NH}, \mathrm{NH}_{2}\right), 3055\left(\mathrm{CH}\right.$, aromatic), $2986\left(\mathrm{CH}_{3}\right), 2220(\mathrm{CN})$, $1688(\mathrm{C}=\mathrm{O}), 1632(\mathrm{C}=\mathrm{C}) ;{ }^{1} \mathrm{H}$ NMR $\left(\mathrm{DMSO}-d_{6}, 300 \mathrm{MHz}\right): \delta=2.78\left(\mathrm{~s}, 3 \mathrm{H}, \mathrm{CH}_{3}\right), 4.98(\mathrm{~s}, 2 \mathrm{H}$, $\mathrm{D}_{2} \mathrm{O}$ exchangeable, $\left.\mathrm{NH}_{2}\right), 5.12,6.46(2 \mathrm{~s}, 2 \mathrm{H}$, pyran $\mathrm{H}-4$, thiazole $\mathrm{H}-5), 7.24-7.52(\mathrm{~m}, 10 \mathrm{H}$, $\left.2 \mathrm{C}_{6} \mathrm{H}_{5}\right), 8.29\left(\mathrm{~s}, 1 \mathrm{H}, \mathrm{D}_{2} \mathrm{O}\right.$ exchaneable, $\left.\mathrm{NH}\right), 10.22\left(\mathrm{~s}, 1 \mathrm{H}, \mathrm{D}_{2} \mathrm{O}\right.$ exchangeable, $\left.\mathrm{OH}\right) ;{ }^{13} \mathrm{C}$ NMR (DMSO- $\left.d_{6}, 75 \mathrm{MHz}\right): \delta 36.8\left(\mathrm{CH}_{3}\right), 51.6$ (pyran C-4), $116.9(\mathrm{CN}), 120.5,121.8,122.6,123.9$ 124.5, 125.1,125.3, $126.6\left(2 \mathrm{C}_{6} \mathrm{H}_{5}\right), 131.8,132.6,133.0,134.7,135.2,142.9$ (pyran, thiazole $\mathrm{C}$ ), 166.6 $(\mathrm{C}=\mathrm{N}), 166.5(\mathrm{C}=\mathrm{O})$. Anal. calcd. for $\mathrm{C}_{23} \mathrm{H}_{19} \mathrm{~N}_{5} \mathrm{O}_{3} \mathrm{~S}(445.49)$ : $\mathrm{C}, 62.01 ; \mathrm{H}, 4.30 ; \mathrm{N}, 15.72$; S, 7.20\%. Found: C, 61.80; H, 4.41; N, 15.92; S, 7.35\%. MS: m/z $445\left[\mathrm{M}^{+}, 28 \%\right]$.

2-Amino-5-(3,5-dihydroxy-1H-pyrazole-1-carbonyl)-6-methyl-4-phenyl-4H-pyran-3-carbonitrile (19). To a solution of compound $6(2.70 \mathrm{~g}, 0.01 \mathrm{~mol})$ in 1,4-dioxane $(40 \mathrm{~mL})$ diethylmalonate $(1.60 \mathrm{~g}, 0.01 \mathrm{~mol})$ was added. The reaction mixture was heated under reflux for $3 \mathrm{~h}$ then was left to cool. The reaction mixture was evaporated under vacuum and the remaining product was triturated with ethanol and the formed solid product was collected by filtration. Brown crystals from 1,4-dioxane, yield $(2.44 \mathrm{~g}, 72 \%)$, mp $43-45^{\circ} \mathrm{C}$. IR ( $\left.\mathrm{KBr}\right) v \operatorname{max~cm}{ }^{-1}: 3571,3342\left(\mathrm{OH}, \mathrm{NH}_{2}\right)$, $3057\left(\mathrm{CH}\right.$, aromatic), $2986\left(\mathrm{CH}_{3}\right), 2220(\mathrm{CN}), 1689(\mathrm{C}=\mathrm{O}), 1630(\mathrm{C}=\mathrm{C}) ;{ }^{1} \mathrm{H}$ NMR (DMSO- $d_{6}$, $300 \mathrm{MHz}): \delta=2.72\left(\mathrm{~s}, 3 \mathrm{H}, \mathrm{CH}_{3}\right), 4.81\left(\mathrm{~s}, 2 \mathrm{H}, \mathrm{D}_{2} \mathrm{O}\right.$ exchangeable, $\left.\mathrm{NH}_{2}\right), 5.12,6.58(2 \mathrm{~s}, 2 \mathrm{H}$, pyran $\mathrm{H}-4$ pyrazole $\mathrm{H}-4), 7.23-7.48\left(\mathrm{~m}, 5 \mathrm{H}, \mathrm{C}_{6} \mathrm{H}_{5}\right), 10.30,10.36(2 \mathrm{~s}, 2 \mathrm{H}, 2 \mathrm{OH}) ;{ }^{13} \mathrm{C}$ NMR (DMSO- $d_{6}$, $75 \mathrm{MHz}): \delta 36.3\left(\mathrm{CH}_{3}\right), 51.2$ (pyran C-4), $116.9(\mathrm{CN}), 120.1,121.4,123.9,124.8\left(\mathrm{C}_{6} \mathrm{H}_{5}\right), 131.2$, 132.6, 133.7, 134.2, 137.3, 141.5 (pyran, pyrazole $\mathrm{C}), 164.6(\mathrm{C}=\mathrm{N}), 167.8(\mathrm{C}=\mathrm{O})$. Anal. calcd. for $\mathrm{C}_{17} \mathrm{H}_{14} \mathrm{~N}_{4} \mathrm{O}_{4}$ (338.32): C, 60.35; H, 4.17; N, 16.56\%. Found: $\mathrm{C}, 60.49 ; \mathrm{H}, 4.36 ; \mathrm{N}, 16.72 \%$. MS: $\mathrm{m} / \mathrm{z} 338\left[\mathrm{M}^{+}, 60 \%\right]$.

6-Amino-5-cyano-2-methyl-4-phenyl-N'-(1-phenylethylidene)-4H-pyran-3-carbohydrazide (21). To a solution of compound $6(2.70 \mathrm{~g}, 0.01 \mathrm{~mol})$ in 1,4-dioxane $(40 \mathrm{~mL})$ acetophenone $(1.20 \mathrm{~g}$, $0.01 \mathrm{~mol}$ ) was added. The reaction mixture was heated under reflux for $1 \mathrm{~h}$ then was left to cool. The reaction mixture was evaporated under vacuum and the remaining product was triturated with ethanol and the formed solid product was collected by filtration. Brown crystals from ethanol, yield $(2.45 \mathrm{~g}, 66 \%), \mathrm{mp} 65-68{ }^{\circ} \mathrm{C}$. IR $(\mathrm{KBr}) v \operatorname{max~cm}{ }^{-1}: 3462-3358\left(\mathrm{NH}, \mathrm{NH}_{2}\right), 3055(\mathrm{CH}$, aromatic), $2972\left(\mathrm{CH}_{3}\right), 2222(\mathrm{CN}), 1689(\mathrm{C}=\mathrm{O}), 1632(\mathrm{C}=\mathrm{C}) ;{ }^{1} \mathrm{H}$ NMR (DMSO- $\left.d_{6}, 300 \mathrm{MHz}\right): \delta$ $=2.71,2.86\left(2 \mathrm{~s}, 6 \mathrm{H}, 2 \mathrm{CH}_{3}\right), 4.93\left(\mathrm{~s}, 2 \mathrm{H}, \mathrm{D}_{2} \mathrm{O}\right.$ exchangeable, $\left.\mathrm{NH}_{2}\right), 5.10(\mathrm{~s}, 1 \mathrm{H}$, pyran $\mathrm{H}-4), 7.21-$ $7.49\left(\mathrm{~m}, 10 \mathrm{H}, 2 \mathrm{C}_{6} \mathrm{H}_{5}\right), 8.40\left(\mathrm{~s}, 1 \mathrm{H}, \mathrm{D}_{2} \mathrm{O}\right.$ exchangeable, $\left.\mathrm{OH}\right) ;{ }^{13} \mathrm{C}$ NMR (DMSO- $\left.d_{6}, 75 \mathrm{MHz}\right): \delta$ 36.4, $38.8\left(2 \mathrm{CH}_{3}\right), 51.2$ (pyran C-4), $116.9(\mathrm{CN}), 120.4,122.7,124.3,125.9\left(\mathrm{C}_{6} \mathrm{H}_{5}\right), 132.3,132.6$, 133.8, 134.9, (pyran), $164.6(\mathrm{C}=\mathrm{N}), 168.1(\mathrm{C}=\mathrm{O})$. Anal. calcd. for $\mathrm{C}_{22} \mathrm{H}_{20} \mathrm{~N}_{4} \mathrm{O}_{2}(372.42)$ : $\mathrm{C}, 70.95$; H, 5.41; N, 15.04\%. Found: C, 71.26; H, 5.55; N, 14.82\%. MS: m/z 372 [M+1, 80\%]. 
6-Amino-5-cyano-2-methyl-4-phenyl-4H-pyran-3-carboxylic acid (22). To a solution of compound $6(2.70 \mathrm{~g}, 0.01 \mathrm{~mol})$ in ethanol $(40 \mathrm{~mL})$ sodium hydroxide $(5 \mathrm{~mL}, 10 \%)$ solution was added. The reaction mixture was heated under reflux for $4 \mathrm{~h}$ then was poured onto ice/water containing hydrochloric acid (till $\mathrm{pH} 6$ ) and the formed solid product was collected by filtration. Pale brown crystals from ethanol, yield $(1.92 \mathrm{~g}, 75 \%), \mathrm{mp} 180-182{ }^{\circ} \mathrm{C}$. IR $(\mathrm{KBr}) v$ max cm${ }^{-1}$ : 3580-3328 (OH, NH$), 3055\left(\mathrm{CH}\right.$ aromatic), $2965\left(\mathrm{CH}_{3}\right), 2220(\mathrm{CN}), 1693(\mathrm{C}=\mathrm{O}), 1630(\mathrm{C}=\mathrm{C})$; ${ }^{1} \mathrm{H}$ NMR (DMSO- $\left.d_{6}, 300 \mathrm{MHz}\right): \delta=2.59\left(\mathrm{~s}, 3 \mathrm{H}, \mathrm{CH}_{3}\right), 4.72\left(\mathrm{~s}, 2 \mathrm{H}, \mathrm{D}_{2} \mathrm{O}\right.$ exchangeable, $\left.\mathrm{NH}_{2}\right)$, 5.13 (s, 1H, pyran $\mathrm{H}-4), 7.25-7.41\left(\mathrm{~m}, 5 \mathrm{H}, \mathrm{C}_{6} \mathrm{H}_{5}\right), 10.22\left(\mathrm{~s}, 1 \mathrm{H}, \mathrm{D}_{2} \mathrm{O}\right.$ exchangeable, $\left.\mathrm{OH}\right) ;{ }^{13} \mathrm{C}$ NMR (DMSO- $\left.d_{6}, 75 \mathrm{MHz}\right): \delta 36.5\left(\mathrm{CH}_{3}\right), 51.3$ (pyran C-4), $116.8(\mathrm{CN}), 120.2,121.6,123.9$, $126.2\left(\mathrm{C}_{6} \mathrm{H}_{5}\right), 132.2,134.3,136.1,138.6$ (pyran $\left.\mathrm{C}\right), 164.5(\mathrm{C}=\mathrm{O})$. Anal. calcd. for $\mathrm{C}_{14} \mathrm{H}_{12} \mathrm{~N}_{2} \mathrm{O}_{3}$ (256.26): C, 65.62; H, 4.72; N, 10.93\%. Found: C, 65.72; H, 4.90; N, 10.75\%. MS: m/z $256\left[\mathrm{M}^{+}\right.$, $42 \%]$.

General procedure for the synthesis of the arylhydrazone derivatives $24 \boldsymbol{a}, \boldsymbol{b}$. To a cold solution of compound $6(2.70 \mathrm{~g}, 0.01 \mathrm{~mol})$ in ethanol $(60 \mathrm{~mL})$ containing sodium hydroxide $(10 \mathrm{~mL}, 10 \%)$ either of benzenediazonium chloride $(0.01 \mathrm{~mol})$ or 4-chlorobenzenediazonium chloride $(0.01 \mathrm{~mol})$ [prepared by the addition of sodium nitrite solution $(1.70 \mathrm{~g}, 0.02 \mathrm{~mol}$ dissolved in water, $10 \mathrm{~mL}$ ) to a cold solution of either aniline $(0.92 \mathrm{~g}, 0.01 \mathrm{~mol})$ or 4-chloroaniline $(1.26 \mathrm{~g}, 0.01 \mathrm{~mol})$ dissolved in concentrated hydrochloric acid $(10 \mathrm{~mL}, 12 \mathrm{M})$ with continuous stirring] was added with continuous stirring. The reaction mixture, in each case, was stirred at room temperature for an additional two hours and the formed solid product, was collected by filtration.

6-Amino-5-cyano-4-phenyl-2-((2-phenylhydrazineylidene)-methyl)-4H-pyran-3-carbohydrazide (24a). Deep red crystals from ethanol, yield $(2.81 \mathrm{~g}, 75 \%), \mathrm{mp} 80-82{ }^{\circ} \mathrm{C}$. IR $(\mathrm{KBr}) v \operatorname{max~cm}{ }^{-1}$ : 3495-3362 (NH, NH$), 3055\left(\mathrm{CH}\right.$ aromatic), $2965\left(\mathrm{CH}_{3}\right), 2222(\mathrm{CN}), 1688(\mathrm{C}=\mathrm{O}), 1632(\mathrm{C}=\mathrm{C})$; ${ }^{1} \mathrm{H}$ NMR (DMSO- $\left.d_{6}, 300 \mathrm{MHz}\right): \delta=4.69,5.26\left(2 \mathrm{~s}, 4 \mathrm{H}, \mathrm{D}_{2} \mathrm{O}\right.$ exchangeable, $\left.2 \mathrm{NH}_{2}\right), 5.23(\mathrm{~s}, 1 \mathrm{H}$, $\mathrm{CH}), 5.11(\mathrm{~s}, 1 \mathrm{H}$, pyran $\mathrm{H}-4), 7.23-7.52\left(\mathrm{~m}, 10 \mathrm{H}, 2 \mathrm{C}_{6} \mathrm{H}_{5}\right), 8.22,8.35\left(2 \mathrm{~s}, 2 \mathrm{H}, \mathrm{D}_{2} \mathrm{O}\right.$ exchangeable, $2 \mathrm{NH}) ;{ }^{13} \mathrm{C}$ NMR (DMSO- $\left.d_{6}, 75 \mathrm{MHz}\right): \delta 51.2$ (pyran C-4), $116.6(\mathrm{CN}), 120.5,121.2,121.4,122.0$, 122.7, 123.9, 124.1, $126.8\left(2 \mathrm{C}_{6} \mathrm{H}_{5}\right), 132.6,133.7,136.5,138.2$ (pyran $\left.\mathrm{C}\right), 164.3(\mathrm{C}=\mathrm{O}), 168.3$ $(\mathrm{C}=\mathrm{N})$. Anal. calcd. for $\mathrm{C}_{20} \mathrm{H}_{18} \mathrm{~N}_{6} \mathrm{O}_{2}(374.40)$ : C, 64.16; H, 4.85; N, 22.45\%. Found: $\mathrm{C}, 64.30 ; \mathrm{H}$, 4.62; N, 22.09\%. MS: m/z $374\left[\mathrm{M}^{+}, 56 \%\right]$.

6-Amino-2-((2-(4-chlorophenyl)hydrazineylidene)methyl)-5-cyano-4-phenyl-4H-pyran-3-carbohydrazide (24b). Deep brown crystals from ethanol, yield $(2.85 \mathrm{~g}, 70 \%), \mathrm{mp} 90-93{ }^{\circ} \mathrm{C}$. IR $(\mathrm{KBr})$ v max cm${ }^{-1}$ : 3479-3341 (NH, $\left.\mathrm{NH}_{2}\right), 3055\left(\mathrm{CH}\right.$ aromatic), $2969\left(\mathrm{CH}_{3}\right), 2220(\mathrm{CN}), 1689(\mathrm{C}=\mathrm{O})$, $1632(\mathrm{C}=\mathrm{C}) ;{ }^{1} \mathrm{H}$ NMR (DMSO- $\left.d_{6}, 300 \mathrm{MHz}\right): \delta=4.72,5.31\left(2 \mathrm{~s}, 4 \mathrm{H}, \mathrm{D}_{2} \mathrm{O}\right.$ exchangeable, $\left.2 \mathrm{NH}_{2}\right)$, $5.26(\mathrm{~s}, 1 \mathrm{H}, \mathrm{CH}), 5.13(\mathrm{~s}, 1 \mathrm{H}$, pyran $\mathrm{H}-4), 7.22-7.50\left(\mathrm{~m}, 9 \mathrm{H}, \mathrm{C}_{6} \mathrm{H}_{5}, \mathrm{C}_{6} \mathrm{H}_{4}\right), 8.24,8.38\left(2 \mathrm{~s}, 2 \mathrm{H}, \mathrm{D}_{2} \mathrm{O}\right.$ exchangeable, $2 \mathrm{NH}$ ); ${ }^{13} \mathrm{C}$ NMR (DMSO- $d_{6}, 75 \mathrm{MHz}$ ): $\delta 51.6$ (pyran C-4), $117.1(\mathrm{CN}), 120.3$, 121.6, 122.9, 123.3, 123.7, 124.2, 124.6, $126.1\left(\mathrm{C}_{6} \mathrm{H}_{5}, \mathrm{C}_{6} \mathrm{H}_{4}\right), 132.2,133.4,136.1,138.6$ (pyran C), $164.6(\mathrm{C}=\mathrm{O}), 168.7(\mathrm{C}=\mathrm{N})$. Anal. calcd. for $\mathrm{C}_{20} \mathrm{H}_{17} \mathrm{ClN}_{6} \mathrm{O}_{2}(408.84)$ : C, 58.75; H, 4.19; N, 20.56\%. Found: C, 58.49; H, 4.31; N, 20.80\%. MS: m/z 408 [M+, 60\%].

6-Amino-2-methyl-5-(4-oxo-4,5-dihydrothiazol-2-yl)-4-phenyl-4H-pyran-3-carbohydrazide (26). To a solution of compound $6(2.70 \mathrm{~g}, 0.01 \mathrm{~mol})$ in ethanol $(40 \mathrm{~mL})$ containing triethylamine $(2.0$ $\mathrm{mL})$ ethyl glycolate $(1.20 \mathrm{~g}, 0.01 \mathrm{~mol})$ was added. The reaction mixture was heated under reflux for $4 \mathrm{~h}$ then was poured onto ice/water and the formed solid product was collected by filtration. Brown crystals from ethanol, yield $(2.06 \mathrm{~g}, 60 \%)$, mp 170-172 ${ }^{\circ} \mathrm{C}$. IR $(\mathrm{KBr}) v$ max cm${ }^{-1}: 3480$ $3339\left(\mathrm{NH}, \mathrm{NH}_{2}\right), 3055\left(\mathrm{CH}\right.$, aromatic), $2986\left(\mathrm{CH}_{3}\right), 1702,1689(2 \mathrm{C}=\mathrm{O}), 1632(\mathrm{C}=\mathrm{C}) ;{ }^{1} \mathrm{H}$ NMR (DMSO- $\left.d_{6}, 300 \mathrm{MHz}\right): \delta=2.71\left(\mathrm{~s}, 3 \mathrm{H}, \mathrm{CH}_{3}\right), 3.93\left(\mathrm{~s}, 2 \mathrm{H}\right.$ thiazole $\left.\mathrm{CH}_{2}\right), 4.93,5.11\left(2 \mathrm{~s}, 4 \mathrm{H}, \mathrm{D}_{2} \mathrm{O}\right.$ exchangeable, $\left.2 \mathrm{NH}_{2}\right), 5.10(\mathrm{~s}, 1 \mathrm{H}$, pyran $\mathrm{H}-4), 7.24-7.45\left(\mathrm{~m}, 5 \mathrm{H}, \mathrm{C}_{6} \mathrm{H}_{5}\right), 8.32\left(\mathrm{~s}, 1 \mathrm{H}, \mathrm{D}_{2} \mathrm{O}\right.$ exchangeable, $\mathrm{NH}) ;{ }^{13} \mathrm{C}$ NMR (DMSO- $\left.d_{6}, 75 \mathrm{MHz}\right): \delta 36.6\left(\mathrm{CH}_{3}\right), 51.1$ (pyran C-4), $80.3(\mathrm{~s}, 2 \mathrm{H}$, 
thiazole $\left.\mathrm{CH}_{2}\right), 120.4,121.3,124.6,125.2\left(\mathrm{C}_{6} \mathrm{H}_{5}\right), 132.1,132.4,133.7,134.2($ pyran $), 166.8(\mathrm{C}=\mathrm{N})$, 167.2, $168.8(2 \mathrm{C}=\mathrm{O})$. Anal. calcd. for $\mathrm{C}_{16} \mathrm{H}_{16} \mathrm{~N}_{4} \mathrm{O}_{3} \mathrm{~S}$ (344.39): C, 55.80; H, 4.68; N, 16.27; $\mathrm{S}$, 9.31\%. Found: C, 55.59; H (4.71; N, 16.39; S, 9.25\%. MS: m/z 344 [M+, 72\%].

\section{CONCLUSION}

Throughout this work we aimed to study the effect of electronegative substituents to either aryl group and/or heterocyclic ring for inhibitions toward cancer cell lines. The target molecules were synthesised using ethyl acetoacetate. Their structures were confirmed by multiple techniques and the synthesized compounds were screened for cytotoxic activity against a panel of six human cancer cell lines using MTT assay. The produced compounds tested against cancer six cancer cell lines and showed that compounds 8b, 10b, 11a, 17a, 21 and 24a were the most cytotoxic compounds. Further tests of the latter compounds toward the five tyrosine kinases c-Kit, Flt-3, VEGFR-2, EGFR, and PDGFR and Pim-1 kinase showed that compounds 10b, 21 and 24a were the most potent of the tested compounds and compounds 10a, 11a and 17a were of the highest inhibitions toward Pim-1 kinase

\section{REFERENCES}

1. Dömling, A. Recent developments in isocyanide based multicomponent reactions in applied chemistry. Chem. Rev. 2006, 106, 17-89.

2. Rivera, D.G.; León, F.; Concepción, O.; Morales, F.E.; Wessjohann, L.A. A multiple multicomponent approach to chimeric peptide-peptoid podands. Chem. Eur. J. 2013, 19, 6417-6428.

3. Ugi, I.; Werner, B.; Dömling, A. The chemistry of isocyanides, their multicomponent reactions and their libraries. Molecules 2003, 8, 53-66.

4. Berkel, S.S.; Bögels, B.G.; Wijdeven, M.A.; Westermann, B.; Rutjes, F.P. Recent advances in asymmetric isocyanide-based multicomponent reactions. Eur. J. Org. Chem. 2012, 2012, 3543-3559.

5. Bonsignore, L.; Loy, G.; Secci, D.; Calignano, A. Synthesis and pharmacological activity of 2-oxo-(2H) 1-benzopyran-3-carboxamide derivatives. Eur. J. Med. Chem. 1993, 28, 517-520.

6. Hafez, E.A.A.; Elnagdi, M.H.; Elagamey, A.G.A.; El-Taweel, F.M.A.A. Nitriles in heterocyclic synthesis: novel synsthesis of benzo $[c]$-coumarin and of benzo $[c]$ pyrano $[3,2-$ c] quinoline derivatives. Heterocycles 1987, 26, 903-907.

7. Darbarwar, M.; Sundaramurthy, V. Synthesis of coumarins with 3:4-fused ring systems and their physiological activity. Synthesis 1982, 337-338.

8. Kemnitzer, W.; Drewe, J.; Jiang, S.; Zhang, H.; Wang, Y.; Zhao, J.; Jia, S.; Herich, J.; Labreque, D.; Storer, R.; Meerovitch, K.; Bouffard, D.; Rej, R.; Denis, R.; Blais, C.; Lamothe, S.; Attardo, G.; Gourdeau, H.; Tseng, B.; Kasibhatla, S.; Cai, S.X. Discovery of 4-aryl-4Hchromenes as a new series of apoptosis inducers using a cell- and caspase-based highthroughput screening assay. 1. Structure-activity relationships of the 4-aryl group. J. Med. Chem. 2004, 47, 6299-6310.

9. Mohareb, R.M.; Al-fourouk, F.O.; Wardakhan, W.W. Uses of dimedone for the synthesis of new heterocyclic derivatives with anti-tumor, c-Met, tyrosine, and Pim-1 kinases inhibitions. Med. Chem. Res. 2018, 27, 1984-2003.

10. Mohareb, R.M.; Al-Omran, F.; Ibrahim, R.A. The uses of cyclohexan-1,4-dione for the synthesis of thiophene derivatives as new anti-proliferative, prostate anticancer, c-Met and tyrosine kinase inhibitors. Med. Chem. Res. 2018, 27, 618-633.

11. Mohareb, R.M.; Hilmy, K.M.H.; Elshehawy, Y.A. Discovery of new thiophene, pyrazole, isoxazole derivatives as antitumor, c-Met, tyrosine kinase and Pim-1 kinase inhibitors. Bull. Chem. Soc. Ethiop. 2018, 32, 285-308. 
12. Yahya, S.M.M.; Elmegeed, G.A.; Mohamed, M.S.; Mohareb, R.M.; Abd-Elhalim, M.M.; Elsayed; G.H. The effect of newly synthesized heterosteroids on miRNA34a, 98, and 214 expression levels in MCF-7 breast cancer cells. Ind. J. Clin. Biochem. 2018, 33, 328-333.

13. Mohareb, R.M.; Halim, P.A. Uses of anthranilic acid for the synthesis of dihydroquinazolin derivatives with antitumor, antiproliferative and Pim-1 kinase activities. Acta Chem Slov. 2018, 65, 554-568.

14. Loncle, C.; Brunel, J.M.; Vidal, N.; Dherbomez, M.; Letourneux, Y. Synthesis and antifungal activity of cholesterol-hydrazone derivatives. Eur. J. Med. Chem. 2004, 39, 1067-1071.

15. Küçükgüzel, S.G.; Mazi, A.; Sahin, F.; Öztürk, S.; Stables, J. Synthesis and biological activities of diflunisal hydrazide-hydrazones. Eur. J. Med. Chem. 2003, 38, 1005-1013.

16. Todeschini, A.R.; Miranda, A.L.P.; Silva, K.C.M.; Parrini, S.C.; Barreiro, E. Synthesis and evaluation of analgesic, antiinflammatory and antiplatelet properties of new 2pyridylarylhydrazone derivatives. Eur. J. Med. Chem. 1998, 33, 189-199.

17. Melnyk, P.; Leroux, V.; Sergheraert, C.; Grellier, P. Design, synthesis and in vitro antimalarial activity of an acylhydrazone library. Bioorg. Med. Chem. Lett. 2006, 16, 31-35.

18. Leite, L.F.C.C.; Ramos, M.N.; da Silva, J.B.P.; Miranda, A.L.P.; Fraga, C.A.M.; Barreiro, E.J. Synthesis and analgesic profile of novel N-containing heterocycle derivatives: arylidene 3-phenyl-1,2,4-oxadiazole-5-carbohydrazide. Il Farmaco 1999, 54, 747-757.

19. Lima, P.C.; Lima, L.M.; da Silva, K.C.M.; Léda, P.H.O.; Miranda, A.L.P.; Fraga, C.A.M.; Barreiro, E.J. Synthesis and analgesic activity of novel N-acylarylhydrazones and isosters, derived from natural safrole. Eur. J. Med. Chem. 2000, 35, 187-203.

20. Cunha, A.C.; Figueiredo, J.M.; Tributino, J.L.M.; Miranda, A.L.P.; Castro, H.C.; Zingali, R.B.; Fraga, C.A.M.; de Souza, M.C.B.V.; Ferreira, V.F.; Barreiro, E.J. Antiplatelet properties of novel N-substituted-phenyl-1,2,3-triazole-4-acylhydrazone derivatives. Bioorg. Med. Chem. 2003, 11, 2051-2059.

21. Bedia, K.K.; Elçin, O.; Seda, U.; Fatma, K.; Nathaly, S.; Sevim, R.; Dimoglo, A. Synthesis and characterization of novel hydrazide-hydrazones and the study of their structureantituberculosis activity. Eur. J. Med. Chem. 2006, 41, 1253-1261.

22. Mohareb, R.M.; Fleita, D.H.; Sakka, O.K. Novel synthesis of hydrazide-hydrazone derivatives and their utilization in the synthesis of coumarin, pyridine, thiazole and thiophene derivatives with antitumor activity. Molecules 2010, 23, 16-27.

23. Wardakhan, W.W.; El-Sayed, N.N.; Mohareb, R.M. Synthesis and anti-tumor evaluation of novel hydrazide and hydrazide-hydrazone derivatives. Acta Pharm. 2013, 63, 45-57.

24. Angelova, V.T.; Valcheva, V.; Vassilev, N.G. ; Buyukliev, R.; Momekov, G.; Dimitrov, I.; Saso, L.; Djukic, M.; Shivachev, B. Antimycobacterial activity of novel hydrazide-hydrazone derivatives with $2 \mathrm{H}$-chromene and coumarin scaffold. Bioorg. Med. Chem. Lett. 2016, 27, 223-227.

25. Mohareb, R.M.; El-Khair A.A. Novel synthesis of hydrazide-hydrazone and their uses for the synthesis of 1,3,4-oxadiazine, 1,2,4-triazine, pyrazole and pyridazine derivatives with antimicrobial and antifungal activities. Inter. J. Appl. Pharm. Technol. 2011, 2, 435-446.

26. Mohareb, R.M.; Mona M. Kamel, M.M.; Milad, Y.R. Uses of $\beta$-diketones for the synthesis of novel heterocyclic compounds and their antitumor evaluations. Bull. Chem. Soc. Ethiop. 2020, 34, 385-405.

27. Sherif, S.M.; Wardakhan, W.W.; Mohareb, R.M. $\beta$-Enaminonitrilers in heterocyclic sytnthesis: A novel one-pot synthesis of thiophenes and their fused derivatives. J. Chem. Res. 1996, 356; 1996, 1970.

28. Mohareb, R.M.; Zohdi, H.F.; Sherif, S.M.; Wardakhan, W.W. Heterocyclic synthesis with isothiocyanates: An expeditious synthetic route for polyfunctionally substituted 3-(thiazol-2'ylidene)pyridines and their fused derivatives. Tetrahedron 1994, 50, 5807-5820.

29. El-Kousy, S.M.; Mohareb, R.M.; Sherif, S.M. Heterocyclic synthesis with isothiocyanate: an expecditious synthetic route to polyfunctionally substituted thiophene, pyrazole, oxazole, 2,3- 
dihydrothiazole, 2-(pyrazol-4-ylideno)thiazole and 5-(thiazol-2-ylideno)-pyrimidine derivatives . J. Chem. Res. 1993, 312; 1993, 1981.

30. Liu, L.; Siegmund, A.; Xi, N.; Kaplan-Lefko, P.; Rex, K.; Chen, A.; Lin, J.; Moriguchi, J.; Berry, L.; Huang, L.Y.; Teffera, Y.; Yang, Y.J.; Zhang, Y.H.; Bellon, S.F.; Lee, M.; Shimanovich, R.; Bak, A.; Dominguez, C.; Norman, M.H.; Harmange, J.C.; Dussault, I.; Kim, T.S. Discovery of a potent, selective, and orally bioavailable c-Met inhibitor: 1-(2hydroxy-2-methylpropyl)-N-(5-(7-methoxyquinolin-4-yloxy)pyridin-2-yl)-5-methyl-3-oxo2-phenyl-2,3-dihydro-1H-pyrazole-4-carboxamide (AMG 458). J. Med. Chem. 2008, 51, 3688-3691.

31. Peach, M.L.; Tan, N.; Tan, N.; Choyke, S.J.; Giubellino, A.; Athauda, G.; Burke, T.R.; Nicklaus, M.C.; Bottaro, D.P. Directed discovery of agents targeting the Met tyrosine kinase domain by virtual screening. J. Med. Chem. 2009, 52, 943-951.

32. Bacco, F.D.; Luraghi, P.; Medico, E.; Reato, G.; Girolami, F.; Perera, T.; Gabriele, P.; Comoglio, P.M.; Boccaccio, C. Induction of MET by ionizing radiation and its role in radioresistance and invasive growth of cancer. J. Natl. Cancer Inst. 2011, 103, 645-661. 